\title{
FT-IR absorption spectroscopy of BEDT-TTF radical salts: charge transfer and donor-anion interaction
}

\author{
J. Moldenhauer, Ch. Horn, K.I. Pokhodnia* and D. Schweitzer \\ 3. Physikalisches Institut, Universität Stuttgart, Pfaffenwaldring 57, 7000 Stuttgart 80 (Germany) \\ I. Heinen and H.J. Keller \\ Anorganisch-Chemisches Institut, Universität Heidelberg Im Neuenheimer Feld 270, 6900 Heidelberg (Germany)
}

(Received December 23, 1992; in revised form February 12, 1993; accepted February 15, 1993)

\begin{abstract}
IR absorption spectra from various $\kappa$-phases of BEDT-TTF radical cation salts as well as from $\alpha$-(BEDT-TTF $)_{2} \mathrm{I}_{3}$ are presented. From these spectra one special mode $\nu_{27}$ representing the asymmetric ring $\mathrm{C}=\mathrm{C}$ stretching vibration is assigned and the dependence of its frequency from the average charge on the donor molecule is given. The investigation of the metal-insulator phase transition of $\alpha$-(BEDT-TTF $)_{2} \mathrm{I}_{3}$ at $135 \mathrm{~K}$ with respect to this vibrational model $\nu_{27}$ gives strong indication of a charge localization in at least one of the two crystallographic different stacks. Furthermore, we correlate the frequencies of the $\mathrm{CH}_{2}$ stretching modes of a variety of BEDT-TTF radical salts with the superconducting phase transition temperatures. These frequencies are evidently not charge dependent, but significantly reflect the strength of the interaction of the donor molecule with the respective anion. It could be shown that phases with a higher $T_{\mathrm{c}}$ of their superconducting transition exhibit a smaller red shift of these frequencies, i.e., a less attractive donor-anion interaction.
\end{abstract}

\section{Introduction}

In most cases IR spectroscopic investigations of BEDT-TTF radical salts (BEDT-TTF $=$ bis(ethylenedithiolo)tetrathiafulvalene) have been performed using the polarized reflectance technique for a determination of the one-electron band parameters [1-5]. Absorption spectra of powders do not reveal polarization-dependent results, which are important in the field of quasi-twodimensional conductors, but due to the high reflectivity even of thin single crystals it is the only way to obtain high resolution and low noise vibrational spectra. In addition, absorption spectroscopy has its different merits. As for the case of (BEDT-TTF) ${ }_{2} I_{3}$ radical salts, it has been shown [6-8] that different crystallographic phases can be characterized and therefore distinguished precisely by their infrared absorption spectra and that even minor differences below the scale of a unit cell become visible in the $\mathrm{CH}_{2}$ stretching vibration bands.

In the early stages of this work there was some evidence [9] that the interaction between the terminal $\mathrm{CH}_{2}$ groups of the BEDT-TTF donor molecules and the anions (reflecting itself in the frequencies of the said vibrations) covers a wider range than in the iodine

"On leave from the Institute of Semiconductors, Ukrainian Academy of Sciences, Kiev, Ukraine. salts, when taking into account the whole variety of commonly employed anions. Following Whangbo et al. [10], the strength of this interaction plays a dominant role concerning the transition into the superconducting state and therefore the value of $T_{c}$.

For the case of radical salts of the donor BEDOTTF (bis-(ethylenedioxy)tetrathiafulvalene) we have found [11] a dependence of various vibrational frequencies upon the average charge on the respective donor. Concerning the relationship between average charge per donor and several vibrational frequencies, some work has been done for the BEDT-TTF precursor TTF and some of its salts $[12,13]$ and for TMTSF and TMTTF [14], but to our knowledge no results have been reported for BEDT-TTF radical salts except for the work of Kozlov et al. $[15,16]$ on the donor BEDTTTF and its radical cation BEDT-TTF ${ }^{+}$.

An investigation of the weak but mostly observable ring $\mathrm{C}=\mathrm{C}$ stretching mode of symmetry $\mathrm{b}_{1 \mathrm{u}}\left(\nu_{27}\right)$ should give some insight into the dependence of its frequency on the average charge per donor, especially because there have been some speculations on the assignment of strong bands in this frequency range as vibronic bands. These bands are due to a coupling of the radical electron to the molecular vibration (EMV coupling) [17] and their spectroscopic manifestation is well de- 
scribed for the case of quasi-one-dimensional conductors $[18,19]$. Although a theory for the quasi-two-dimensional case is still missing, we will present some candidates for such vibronic bands.

Furthermore, in case of a clear dependence between the frequency of the $\nu_{27}$ mode and the average charge per donor molecule, it is possible to investigate metal-insulator (M-I) transitions such as in $\alpha$-(BEDTTTF) $)_{2} I_{3}$ with respect to a localization of charge.

\section{Experimental}

All investigated radical salts of BEDT-TTF were prepared electrochemically in the standard way except the $1: 1$ salt, (BEDT-TTF) ${ }_{3}$, which was prepared by a direct reaction of the donor with iodine in THF. The presence of triiode anions in the latter was verified by the IR spectrum of the $\mathrm{I}_{3}{ }^{-}$stretching and bending vibrations. For IR absorption measurements, crystals were ground with $\mathrm{KBr}$ in an agate mortar and afterwards pressed to pellets as described in ref. 6 .

The spectra were taken on a commercial Fourier transform spectrometer (BOMEM DA 3.02) with a fast InSb detector in the range $5000-1800 \mathrm{~cm}^{-1}$, an MCT detector between 1800 and $450 \mathrm{~cm}^{-1}$ and an applied resolution of $0.5 \mathrm{~cm}^{-1}$. In order to obtain a good signalto-noise ratio, 2000 interferograms were co-added for each spectrum. Low temperature measurements were carried out in a continuous-flow helium cryostat with $\mathrm{KRS5}$ and $\mathrm{ZnSe}$ windows.

\section{Results}

Vibrational spectra of the neutral donor and the simply charged radical cation have already been investigated and assigned $[15,16]$ by means of a normal coordinate analysis (NCA). These spectra together with the one of $\beta$-(BEDT-TTF) ${ }_{2} I_{3}$ (2:1 salt) are shown in Fig. 1(a) (vibrations of the BEDT-TTF framework) and Fig. 1(b) $\left(\mathrm{CH}_{2}\right.$ stretching vibrations). The frequencies on which we will focus our attention are given in Table 1. It should be mentioned that these and all following spectra of $\mathrm{CH}_{2}$ stretching vibrations of radical cation salts are baseline corrected, because these bands usually appear on a wing of a broad electronic absorption extending from about $6000 \mathrm{~cm}^{-1}$ to lowest frequencies [6].

In Fig. 1(b) one can immediately see that the frequencies of $\mathrm{CH}_{2}$ stretching vibrations are nearly unaffected by the respective charge transfer. In contrast, the frequencies assigned to stretching vibrations of the inner and ring $\mathrm{C}=\mathrm{C}$ bonds (see Table 1 ) are very sensitive to a transfer of electrons from the HOMO.
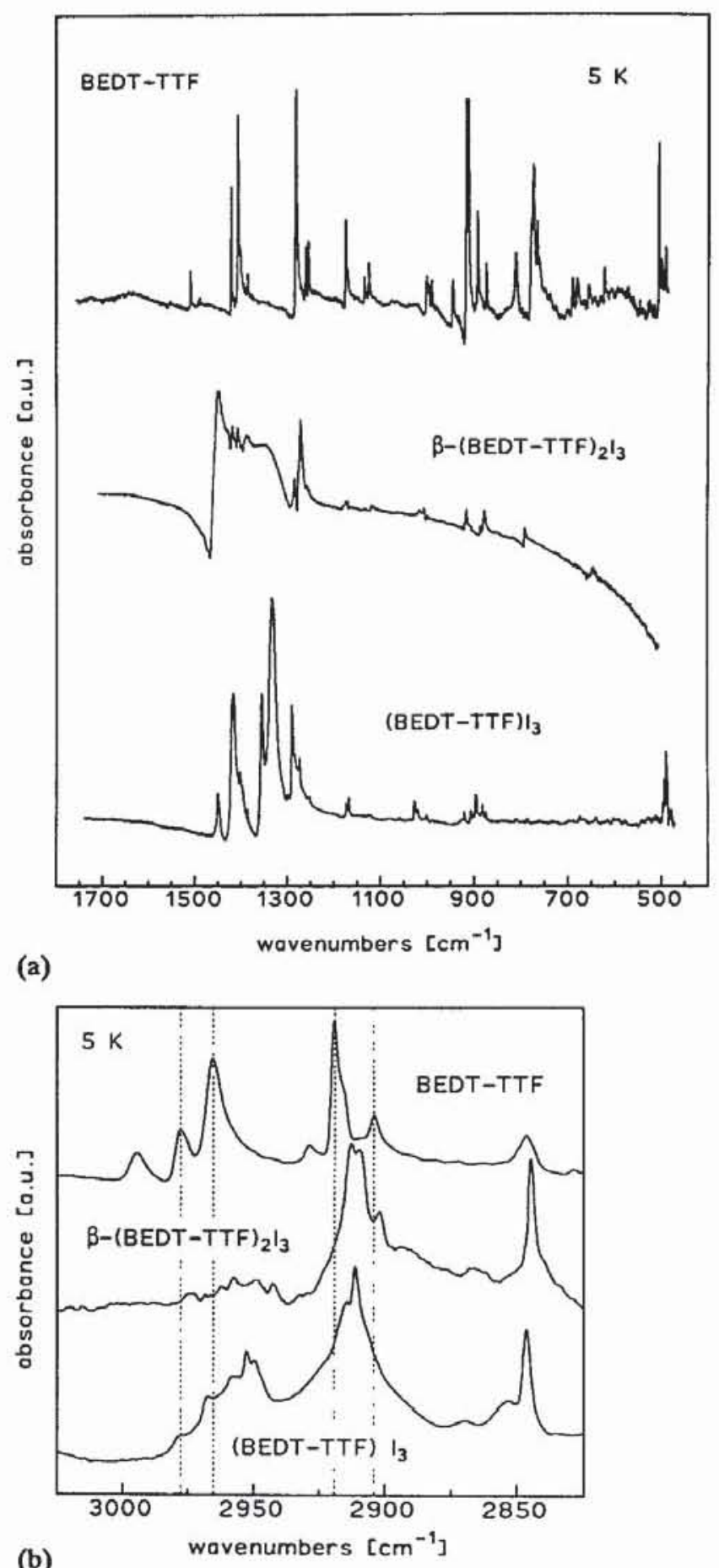

Fig. 1. (a) Absorption spectra of neutral BEDT-TTF, $\beta$-(BEDTTTF) $)_{2} I_{3}$ and (BEDT-TTF) $I_{3}$ at $5 \mathrm{~K}$ in the range from 500 to $1700 \mathrm{~cm}^{-1}$. (b) $\mathrm{CH}_{2}$ stretching vibration bands of neutral BEDTTTF, $\beta$-(BEDT-TTF $)_{2} \mathrm{I}_{3}$ and (BEDT-TTF) $\mathrm{I}_{3}$ at $5 \mathrm{~K}$.

The shift of the IR active mode $\nu_{27}$ displayed in Fig. 2 is due to this charge transfer and is consistent with the results of the NCA in refs. 15 and 16.

As expected for dielectrics, the baselines of the spectra of the insulator BEDT-TTF and the semiconductor BEDT-TTF ${ }^{+} \mathrm{I}_{3}{ }^{-}$are flat (Fig. 1(a)), whereas the $\beta$ phase is a metal and the vibrational features are superimposed on the broad electronic absorption mentioned above with its maximum around $3000 \mathrm{~cm}^{-1}$. 
TABLE 1. Frequencies of $\mathrm{C}=\mathrm{C}$ and $\mathrm{CH}_{2}$ stretching vibrations in the neutral donor BEDT-TTF, the $2: 1$ salt $\beta$-(BEDT-TTF) ${ }_{2} \mathrm{I}_{3}$ and the $1: 1$ salt (BEDT-TTF) $I_{3}$

\begin{tabular}{llll}
\hline Vibration & BEDT-TTF & $\beta$-(BEDT-TTF) $)_{2} \mathrm{I}_{3}$ & (BEDT-TTF) $\mathrm{I}_{3}$ \\
\hline$\nu_{2}\left(\mathrm{a}_{\varepsilon}\right)$ & $1551 \mathrm{Ra}^{\mathrm{a}}$ & $1489 \mathrm{Ra}^{\mathrm{b}}$ & $1455 \mathrm{Ra}^{\mathrm{c}}$ \\
$\nu_{27}\left(\mathrm{~b}_{1 \mathrm{u}}\right)$ & $1508 \mathrm{IR}^{\mathrm{d}}$ & $1473 \mathrm{IR}^{\mathrm{d}}$ & $1449 \mathrm{IR}^{\mathrm{d}}$ \\
$\nu_{3}\left(\mathrm{a}_{2}\right)$ & $1493 \mathrm{Ra}^{\mathrm{d}}$ & $1464 \mathrm{Ra}^{\mathrm{b}}$ & $1431 \mathrm{Ra}^{\mathrm{c}}$ \\
$\mathrm{CH}_{2}$ stretching & $2987 \mathrm{vw}$ & $2973 \mathrm{vww}$ & $2975 \mathrm{vww}$ \\
$\left(\mathrm{b}_{1 u}, \mathrm{~b}_{2 u}, \mathrm{~b}_{3 u}\right)$ & $2965 \mathrm{vw}$ & $2942 / 2958 \mathrm{vw}$ & $2955 / 2965 \mathrm{vw}$ \\
\hline
\end{tabular}

"Kozlov et al. [15]. $\quad$ 'Zamboni et al. [23]. 'Kozlov et al. [16]. ' 'This work.

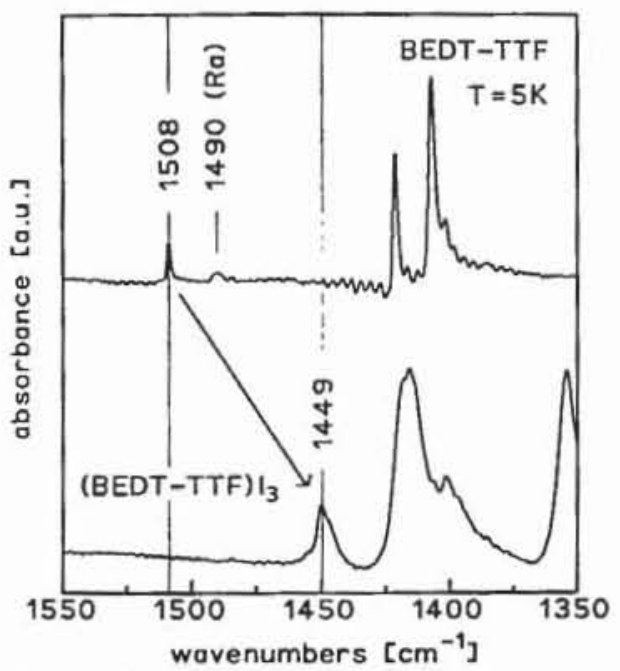

Fig. 2. Shift of the ring $\mathrm{C}=\mathrm{C}$ stretching mode $\nu_{2}\left(\mathrm{~b}_{\mathrm{tu}}\right)$ upon ionization of BEDT-TTF to BEDT-TTF ${ }^{+}$at $5 \mathrm{~K}$.

One should also notice the strong peaks in the spectra of the radical salts which are absent in neutral BEDTTTF. In their region of appearance between 1500 and $1300 \mathrm{~cm}^{-1}$ only the mentioned $\mathrm{C}=\mathrm{C}$ stretching mode and the $\mathrm{CH}_{2}$ scissoring mode are found, both of which are still present in the radical salts. In addition it is not likely that they will gain (compared to other $\mathrm{CH}_{2}$ bending modes) such an enormous intensity and suffer shifts in frequency of $50 \mathrm{~cm}^{-1}$ and more. According to their appearance in the spectra of quasi-1D conductors, they are therefore assigned as vibronic bands, which are due to a coupling of the unpaired radical electron to totally symmetric $\left(\mathrm{a}_{\mathrm{g}}\right)$ vibrations of the donor molecule.

In most of the radical salts of BEDT-TTF (e.g. the $\alpha$ - and $\beta$-phases) the donor molecules make up stacks from more or less dimerized units, arranged in a faceto-face manner. The $\kappa$-phases also consist of BEDTTTF dimers, but their nearest neighbour is always rotated by approximately $90^{\circ}$ with respect to the planes of the BEDT-TTF molecules. The salts with the highest known $T_{\mathrm{c}}$ values belong to this phase, e.g., $\kappa$-(BEDT$\mathrm{TTF})_{2} \mathrm{Cu}(\mathrm{NCS})_{2} \quad[20,21]$ and $\kappa$-(BEDT-TTF $)_{2}-$ $\mathrm{Cu}\left[\mathrm{N}(\mathrm{CN})_{2}\right] \mathrm{Br}[22]$.
The spectra of various $\kappa$-phases are given in Fig. 3 . A specific feature of the spectrum of $\kappa$-(BEDT-TTF $)_{2} \mathrm{I}_{3}$ in comparison to other superconducting iodine salts is the relative high-frequency vibronic band at $1467 \mathrm{~cm}^{-1}$ (versus $1454 \mathrm{~cm}^{-1}$ in the $\beta$-phase, both values at $5 \mathrm{~K}$ ) shown in Fig. 4 and, concerning the multiplets between 2900 and $2930 \mathrm{~cm}^{-1}$, the high-frequency $\mathrm{CH}_{2}$ stretching bands (Fig. 3(b)). It should be mentioned that, in spectra of powders, the broad and the complex shape of vibronic bands results from the overlapping of components polarized along different directions in the plane of the BEDT-TTF layers; additionally it may further be complicated by Fano-like interference effects with the electronic absorption due to interband transitions. Other typical vibrations such as the $\mathrm{C}=\mathrm{C}$ (ring) stretching vibration are found to be split into two bands at 1473 and $1478 \mathrm{~cm}^{-1}$ (Fig. 4). This splitting, which is not observed in the $\alpha$ - and $\beta$-phases of (BEDT-TTF) $)_{2} \mathrm{I}_{3}$, might be due to an asymmetry between the two molecules of a dimer.

Turning our attention to the spectra of BEDT-TTF salts with $\mathrm{Cu}(\mathrm{NCS})_{2}$ and $\mathrm{Cu}\left[\mathrm{N}(\mathrm{CN})_{2}\right] \mathrm{Br}$ anions, we find the rough shape of the absorption between 1800 and $500 \mathrm{~cm}^{-1}$ to be different from that of the iodine salts (Fig. 3(a)). It is remarkable that the absorption bands are rather broad even at $5 \mathrm{~K}$, they look more like those of the triiodide salts at $300 \mathrm{~K}$. This also applies to the $\mathrm{CH}_{2}$ stretching frequencies (Fig. 3(b)). In the region of the vibronic bands (Fig. 4) again the weak $\nu_{27}$ mode is found with the expected frequency; in $\kappa$-(BEDTTTF $)_{2} \mathrm{Cu}\left[\mathrm{N}(\mathrm{CN})_{2}\right] \mathrm{Br}$ there is the same splitting as in $\kappa$-(BEDT-TTF $)_{2} \mathrm{I}_{3}$.

Furthermore, the first intense peak at $1467 \mathrm{~cm}^{-1}$ (assigned as vibronic band) appears in the same position in all three salts in Figs. 3(a) and 4. In the copper salts there are additional broad features at somewhat lower wavenumbers. Similar broad bands are observed in the iodine salts around $1330 \mathrm{~cm}^{-1}$ (at room temperature), which are strongly distorted by anti-resonating $\mathrm{CH}_{2}$ wagging modes. This band around 1330 $\mathrm{cm}^{-1}$ is also present in the copper salts, the difference to the triiodide salts lies in the fact that in the latter 


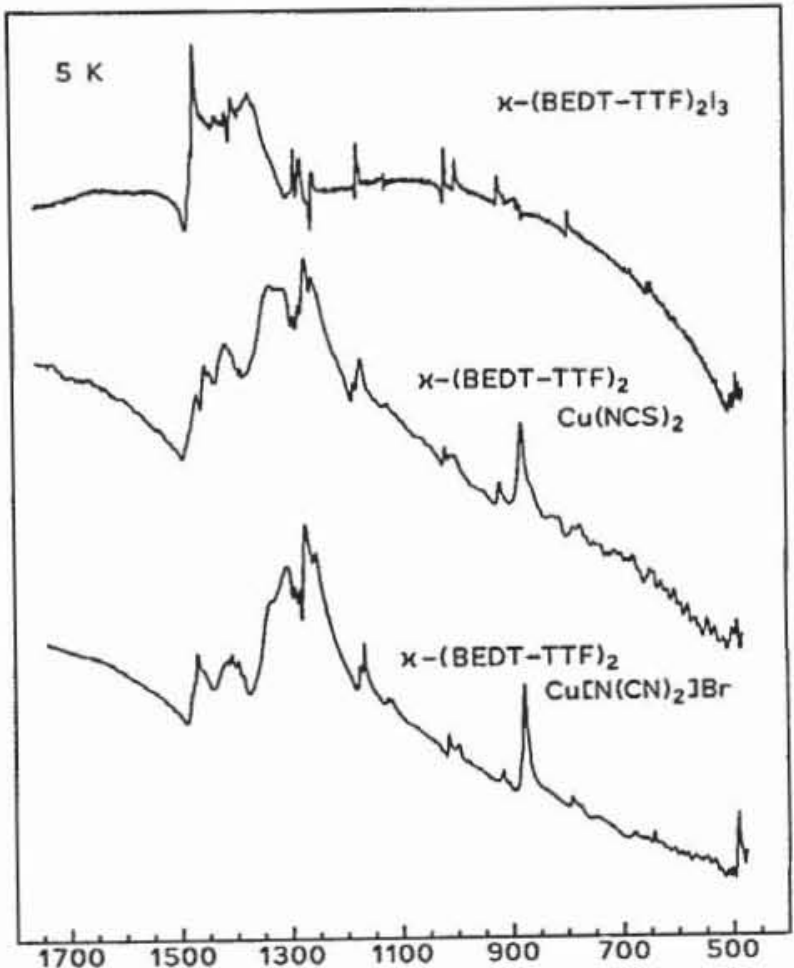

(a)

wavenumbers $\left[\mathrm{cm}^{-1}\right]$

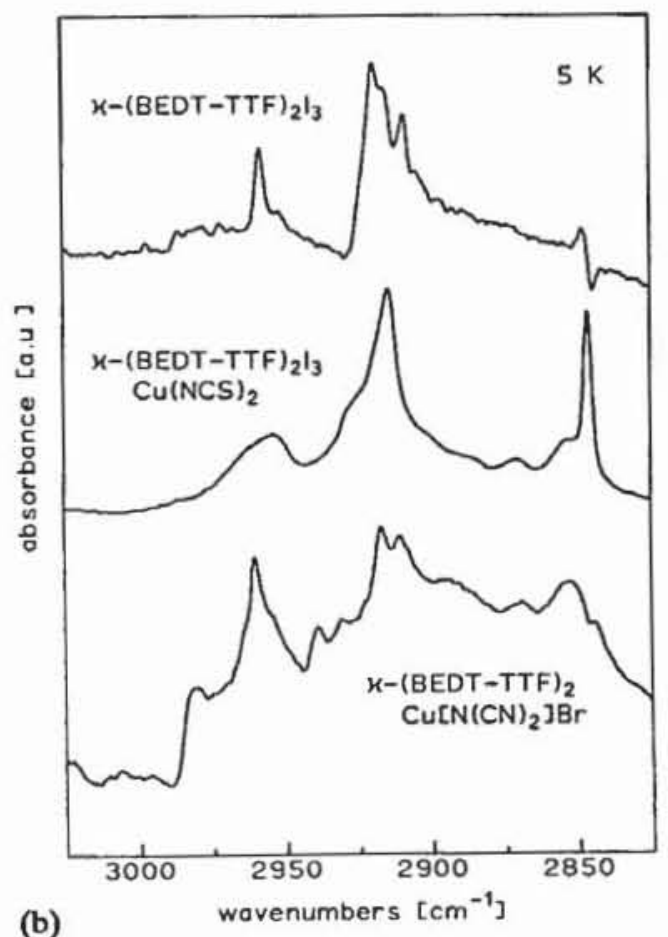

Fig. 3. (a) Absorption spectra of different $\kappa$-phase salts of BEDTTTF at $5 \mathrm{~K}$ in the range from 500 to $1700 \mathrm{~cm}^{-1}$. (b) $\mathrm{CH}_{2}$ stretching vibrations of different $\kappa$-phase salts of BEDT-TTF at $5 \mathrm{~K}$.

it moves upwards about $20 \mathrm{~cm}^{-1}$ on cooling down to $4 \mathrm{~K}$, thereby removing the anti-resonance with the $\mathrm{CH}_{2}$ wagging modes. Another difference between the iodineand the copper-containing salts is that in the former the vibronic bands above $1400 \mathrm{~cm}^{-1}$ are much sharper and more intense than in the latter.

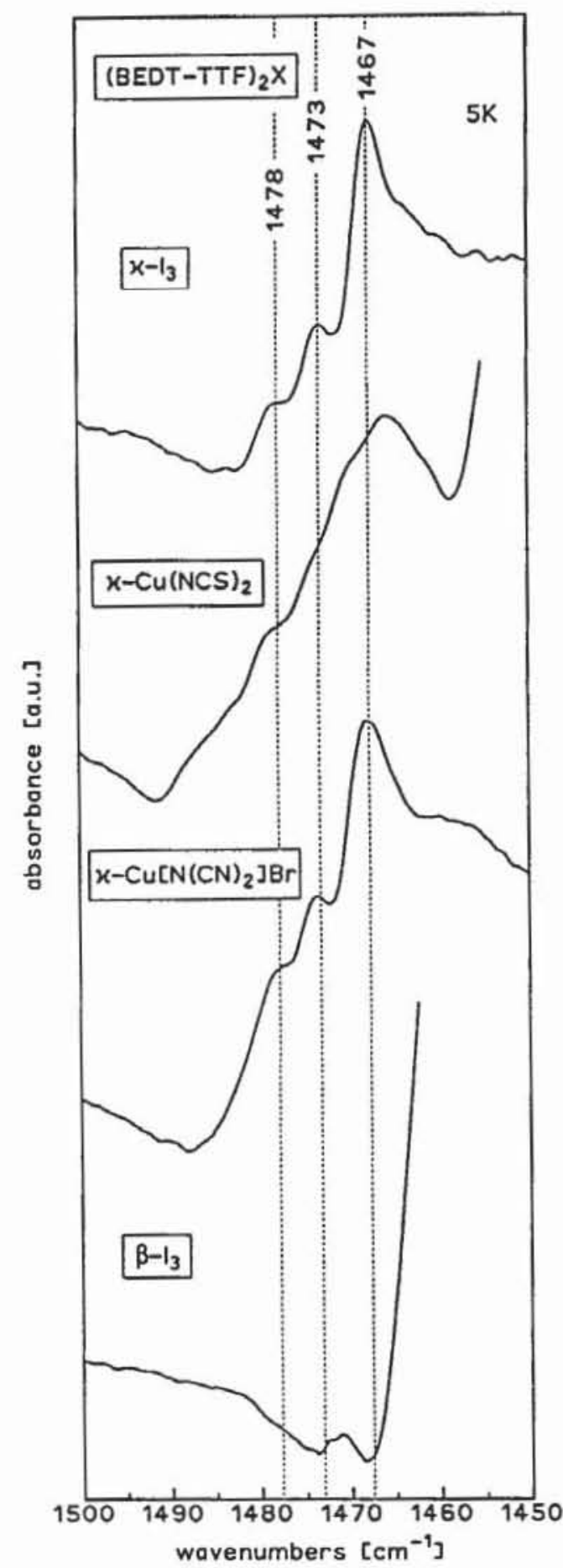

Fig. 4. Absorption spectra of different BEDT-TTF salts in the region of the ring $\mathrm{C}=\mathrm{C}$ stretching vibration $\nu_{2}\left(\mathrm{~b}_{1 \mathrm{u}}\right)$ (see text).

In the investigations on the $\alpha_{1}, \beta-, \kappa^{-}$and $\kappa_{t}$-phases of (BEDT-TTF $)_{2} \mathrm{I}_{3}$ salts [8] no significant temperaturedependent changes concerning the vibrational bands were found aside from a narrowing of bands and continuous small shifts of a few wavenumbers. The situation is different concerning $\alpha$-(BEDT-TTF $)_{2} \mathrm{I}_{3}$, which was expected because of its well-known M-I phase transition at $135 \mathrm{~K}$. While passing the critical temperature, changes can be observed in the region of the vibronic bands and the $b_{14}$ mode $\nu_{27}$ of the ring $\mathrm{C}=\mathrm{C}$ stretching, which are found between 1400 and 


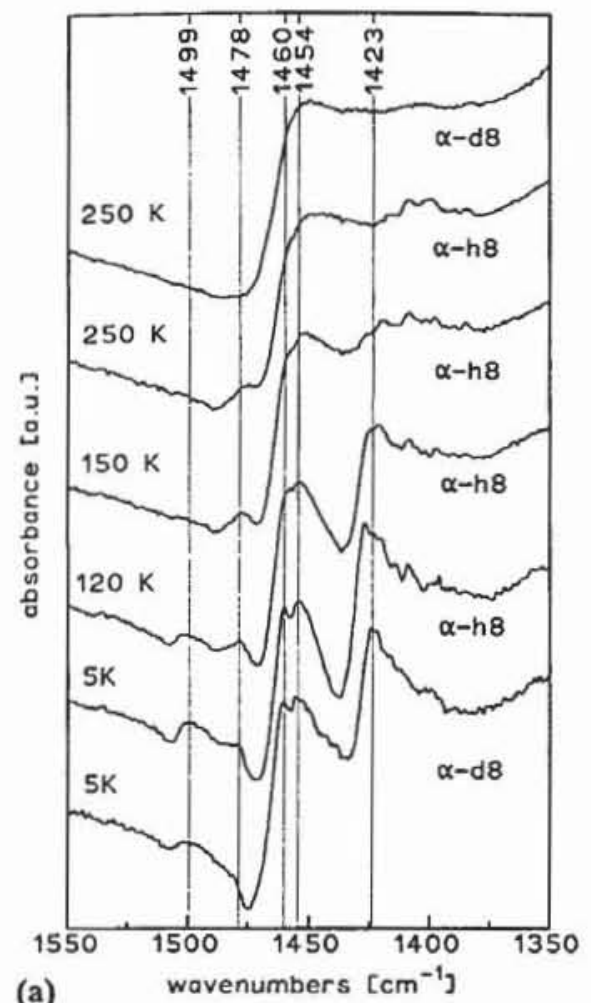

(a)

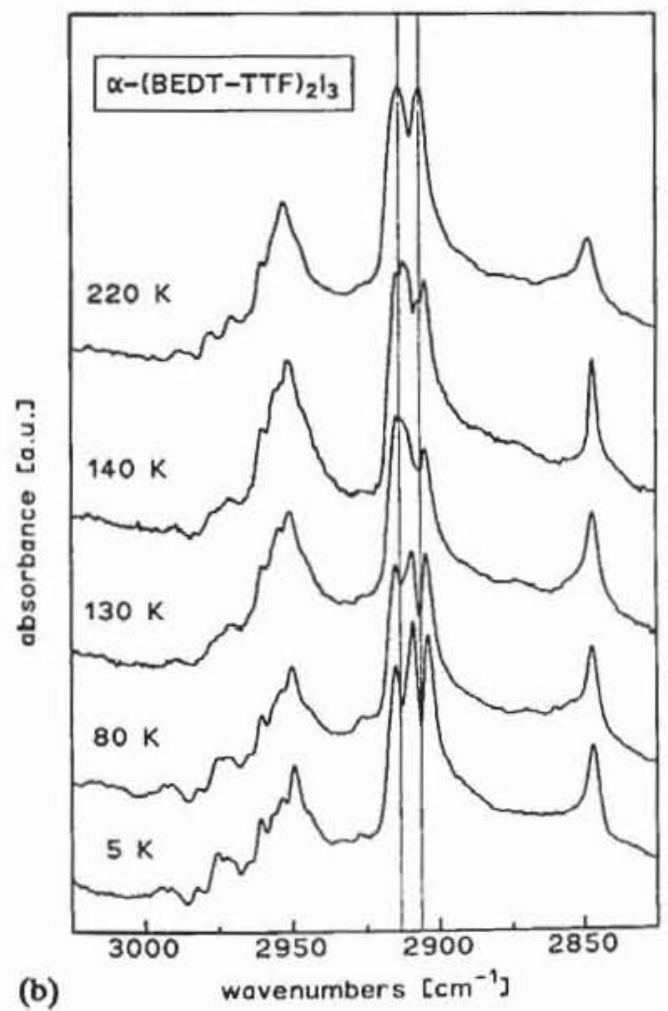

Fig. 5. (a) Absorption bands of $\alpha$-(BEDT-TTF $)_{2} I_{3}$ in the range of the $\mathrm{C}=\mathrm{C}$ stretching vibration at different temperatures ( $\mathrm{h} 8=$ protonated BEDT-TTF, $\mathrm{d} 8=$ deuterated BEDT-TTF). (b) $\mathrm{CH}_{2}$ stretching vibrations of $\alpha$-(BEDT-TTF) $)_{2} \mathrm{I}_{3}$ at different temperatures.

$1500 \mathrm{~cm}^{-1}$. Figure 5(a) shows the spectra of $\alpha$-(BEDTTTF $)_{2} I_{3}$ in this spectral range at several temperatures together with spectra of deuterated $\alpha$-(BEDT-TTF) ${ }_{2} \mathrm{I}_{3}$ $(\alpha-\mathrm{d} 8)$. The comparison of spectra of the protonated and the deuterated salt shows that the mentioned bands have nothing to do with $\mathrm{CH}_{2}$ scissoring modes located around $1420 \mathrm{~cm}^{-1}$.

First, at the temperature of the phase transition, changes occur concerning the strong feature at around $1450 \mathrm{~cm}^{-1}$, which we assign as a vibronic band. Secondly, there are two weak bands emerging in a nearly symmetrical order around the band at $1478 \mathrm{~cm}^{-1}$, namely at 1499 and $1454 \mathrm{~cm}^{-1}$. The poor visibility of the band at $1478 \mathrm{~cm}^{-1}$ in the deuterated compound is due to the fact that here the vibronic band overlaps with the band in question.

On passing through the phase transition the changes in the bands of the $\mathrm{CH}_{2}$ stretching vibrations are minor (Fig. 5(b)). At temperatures slightly above and below the temperature of the M-I phase transition, it is hard to tell changes in the intensities of the bands from small shifts. There are, however, changes and they seem to be related to the changes in the relative positions of BEDT-TTF and iodine molecules because their onset is a little above the phase transition temperature. It might be worth mentioning that these intensity changes proceed on a further decrease of temperature.

\section{Discussion}

In the field of charge transfer salts, which are quasione- or quasi-two-dimensional conductors or semiconductors, there has always been a certain interest in vibrational bands that might reflect the average charge transfer from the donor molecule to the acceptor. Usually it is expected that a decrease of negative charge, i.e., an oxidation of the donor, leads to a decrease of the stretching vibrational frequency of certain bonds in the molecule. In Fig. 2 the frequency shift of the $\mathrm{b}_{1 \mathrm{u}}$ mode of the ring $\mathrm{C}=\mathrm{C}$ groups on taking away one electron from the neutral BEDT-TTF molecule is shown. This mode $\left(\nu_{27}\right)$ together with its totally symmetric pendant $\left(\nu_{3}\right)$ and the totally symmetric mode of the central $\mathbf{C}=\mathbf{C}\left(\nu_{2}\right)$ are given in Table 1 , where results from different papers are given. These modes and the observed frequencies are also displayed in Fig. 6.

Concerning the IR active mode $\nu_{27}$, i.e., the asymmetric stretching vibration of the ring $\mathrm{C}=\mathrm{C}$ groups, we find a good fit of the vibrations of the BEDT$\mathrm{TTF}^{+0.5}$ molecule into a linear dependence of the frequency upon the average charge on the donor molecule. For the sake of clarity the bands of the coppercontaining $\kappa$-phases are not shown here, but from Fig. 4 it can be seen that aside from a splitting they are found $\left( \pm 2 \mathrm{~cm}^{-1}\right)$ in the same position. The Raman band of the related $\mathrm{a}_{\mathrm{g}}$-mode $\nu_{3}$ of the ring $\mathrm{C}=\mathrm{C}$ stretching vibration also exhibits this linear behaviour and the frequency of $\beta$-(BEDT-TTF $)_{2} \mathrm{I}_{3}$ has the expected 


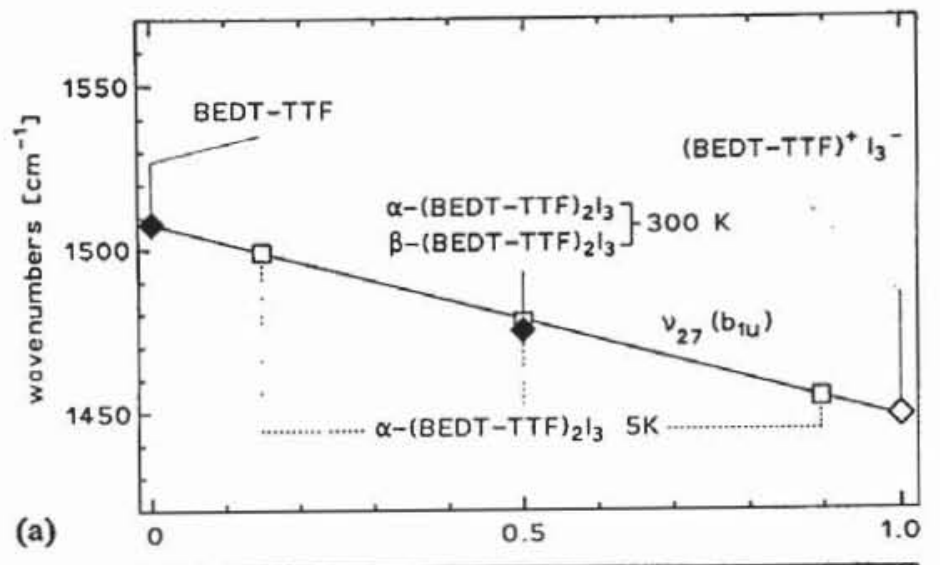

(a)

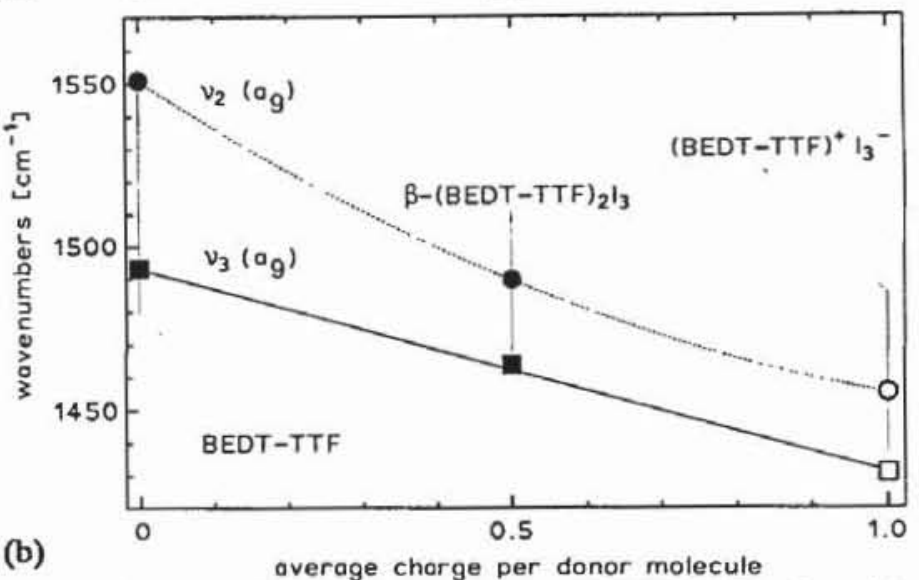

Fig. 6. $\mathrm{C}=\mathrm{C}$ stretching frequencies of BEDT-TTF salts with different degrees of ionization of the donor: (a) IR active mode $\nu_{27}$; (b) Raman active modes $\nu_{2}$ and $\nu_{3}$ (see text).

value. It should be mentioned that the assignment of $\nu_{2}$ and $\nu_{3}$ given in an earlier paper [23] has been exchanged here for evident reasons. The Raman band of the central $\mathrm{C}=\mathrm{C}$ stretching $\left(\nu_{2}\right)$ obviously does not fit into a linear dependence of frequency from the charge on the donor molecule; the reason might be a different behaviour of this bond.

Concerning the frequencies of the vibronic bands, which are expected to appear somewhat below the frequency of the $\nu_{3}$ mode, it should be mentioned that in the investigated $\kappa$-phases (see Fig. 4) the maxima of the bands are nearly in the same position as the $\nu_{3}$ mode. But referring to the restrictions made above concerning the shape of these bands obtained by unpolarized radiation, the position of their maximum might not be very meaningful.

As a result it might be suspected that an IR spectroscopic determination of the average charge transfer from the BEDT-TTF molecules can be performed by looking at the frequency of the ring $\mathrm{C}=\mathrm{C}$ stretching mode $\nu_{27}$. In $\alpha$-(BEDT-TTF) ${ }_{2} \mathrm{I}_{3}$, two new bands emerge at the M-I phase transition that are arranged nearly symmetrical around the $\mathrm{C}=\mathrm{C}$ stretching band at 1477 $\mathrm{cm}^{-1}$, which is still present below the phase transition. The dependence of the frequency $\bar{\nu}$ of the $\nu_{27}$ mode from the average charge $q$ per donor molecule calculated from the frequencies of neutral BEDT-TTF and the charged donor in BEDT-TTF ${ }^{+} \mathrm{I}_{3}{ }^{-}$(see Fig. 1) is of the form:

$$
\vec{\nu}_{27}\left[\mathrm{~cm}^{-1}\right]=-59\left[\frac{\mathrm{cm}^{-1}}{e}\right] \times q[e]+1508\left[\mathrm{~cm}^{-1}\right]
$$

For the frequencies observed below the phase transition the calculated charges are $q=+0.15, q=+0.52$ and $q=+0.9$ (see also Fig. 6). From these results we conclude that a localization of the charge takes place in one of the two crystallographic different stacks of the donor molecules. This is understandable since it was shown by structural investigations [24] that in the stack containing the non-equivalent BEDT-TTF molecules $B$ and $C$ below the phase transition, a dimerization occurs. As mentioned before the $b_{1 u}$ mode at 1477 $\mathrm{cm}^{-1}$ observed above the M-I transition is still present at temperatures below the phase transition. This indicates that in the other stack the charge is still able to move in a way that (on the time scale of the respective frequency of $1500 \mathrm{~cm}^{-1}$ ) one still obtains an average charge of +0.5 on the donor molecules of this stack.

Now even the behaviour of the residual strong bands between 1500 and $1400 \mathrm{~cm}^{-1}$ (Fig. 5(a)), which have been assigned as vibronic bands, becomes clearer. At room temperature only one band slightly above 1450 $\mathrm{cm}^{-1}$ is observed. On decreasing the temperature, it smoothly moves upwards to $1460 \mathrm{~cm}^{-1}$, which might be a real shift or just a change of the slightly antiresonant band shape. In any case the same shift was observed in $\beta$-(BEDT-TTF) ${ }_{2} I_{3}$ from $1440 \mathrm{~cm}^{-1}$ at 300 $\mathrm{K}$ to $1450 \mathrm{~cm}^{-1}$ at $5 \mathrm{~K}$, which exhibits no $\mathrm{M}-\mathrm{I}$ phase transition.

Additionally, below the M-I phase transition temperature, another band at $1423 \mathrm{~cm}^{-1}$ is gaining intensity, which we assign to be due to the dimers of the stack with the localized charge. The frequency of this emerging vibronic band is interesting from the point of the different kinds of dimers that are observed in BEDTTTF radical salts. In 2:1 salts with a metallic behaviour, these vibronic bands are found between 1450 and 1467 $\mathrm{cm}^{-1}$ (at $5 \mathrm{~K}$ ), whereas in various 1:1 salts as in (BEDTTTF) $\mathrm{I}_{3}$ and (BEDT-TTF) $\mathrm{Br}$ this band is located around $1400 \mathrm{~cm}^{-1}$. In phases with a $2: 1$ stoichiometry and a semiconducting behaviour we have found this band around $1425 \mathrm{~cm}^{-1}$, e.g., in $\delta$-(BEDT-TTF) ${ }_{2} \mathrm{AuI}_{2}$ and (BEDT-TTF $)_{2} \mathrm{CuBr}_{2}$. The frequency of this band seems to be strongly dependent not only on the average charge transfer from donor to the anions, but also from the charge distribution upon and between the donor molecules. Therefore, its appearance in $\alpha$-(BEDT-TTF $)_{2} \mathrm{I}_{3}$ below the M-I phase transition is a further indication of the presence of localized charges in one of the two different stacks of the donor molecules. If this conclusion 
is right, it means in addition that the crystals of $\alpha$ $(\text { BEDT-TTF })_{2} \mathrm{I}_{3}$ lose their quasi-two-dimensional electronic properties below the M-I phase transition and exhibit more quasi-one-dimensional properties, since the coupling between neighbouring stacks is lost.

According to Endres et al. [24], at the M-I phase transition, besides a dimerization in one of the two crystallographic different stacks, a slight rotation of the $\mathrm{I}_{3}{ }^{-}$anions takes place. This slight rotation should lead to a change in the donor-anion interaction. The small changes observed in the spectra of $\mathrm{CH}_{2}$ stretching vibrations, which occur near the temperature of the M-I phase transition (Fig. 5(b)), are consistent with this rearrangement.

For the iodine salts of BEDT-TTF, it was already shown [8] that the bands of the $\mathrm{CH}_{2}$ stretching vibrations are characteristic for a certain crystallographic phase. But, furthermore, in these bands there should be at least a qualitative reflection of the strength of the donor-anion interaction, which takes place via $\mathrm{C}-\mathrm{H} \cdot \cdots \mathrm{I}$ contacts and is therefore expected to have the character of a weak hydrogen bond. For simplicity we reduce the term hydrogen bond in the further discussion to an interaction of mainly electrostatic nature, because a hydrogen bond usually requires certain geometric specifications concerning distance and bond angle, i.e., details which cannot be verified completely. Even with these restrictions it is clear that smaller values of the distance $\mathbf{C}-\mathrm{H} \cdots$ anion will result in a stronger bond between donor and anion. The attractive force exerted on the hydrogen atom therefore leads to a stronger red shift of $\mathrm{CH}_{2}$ stretching frequencies.

It has been mentioned earlier that the degree of ionization does not significantly influence the observed frequencies of the latter vibrations (see Fig. 1(b)). This is comprehensible since in NMR investigations [25] at the positions of the ethylene carbons no spin density (and therefore in first order no charge density) is found. Additionally, the following results concern only salts with an average charge of +0.5 per donor molecule. In Fig. 7 we display all observed $\mathrm{CH}_{2}$ stretching frequencies of the relatively strong and most well-resolved bands between 2900 and $2930 \mathrm{~cm}^{-1}$ of several BEDTTTF radical salts against their superconducting transition temperature $T_{\mathrm{c}}$ (the filled symbols represent the arithmetic mean $\langle\bar{\nu}\rangle$ of these frequencies). The solid line is a simple linear fit to the mean values with a slope of $0.9 \mathrm{~cm}^{-1} / \mathrm{K}$; the dashed line is a fit to the highest found frequency with a slope of $1.7 \mathrm{~cm}^{-1} / \mathrm{K}$. The values of the tempered phase $\alpha_{t}$-(BEDT-TTF) ${ }_{2} \mathrm{I}_{3}$, which deviate strongly from the obvious dependence between $\langle\bar{\nu}\rangle$ and $T_{c}$, were left out in this fit because we are not able to tell the 'right' bands from bands which appear due to the presence of neutral BEDTTTF in the tempered crystals. This presence is due to

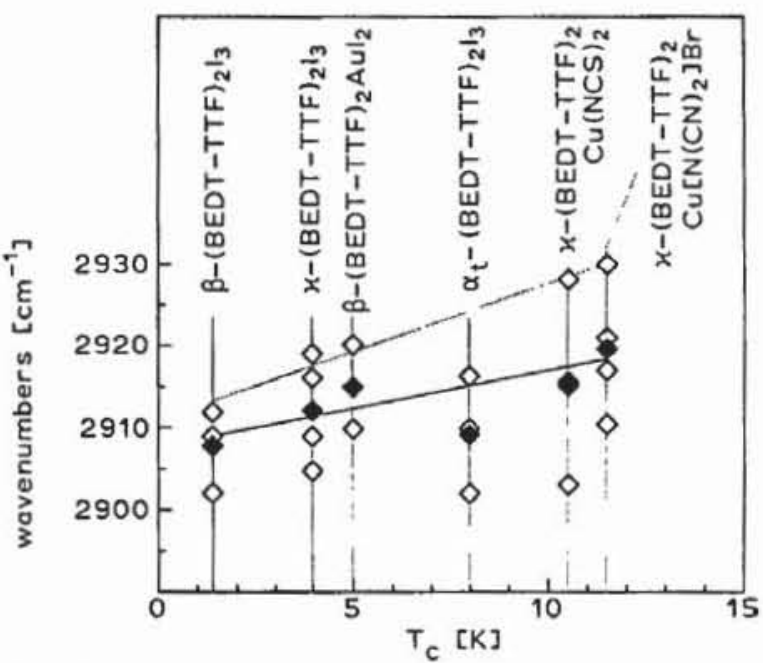

Fig. 7. $\mathrm{CH}_{2}$ stretching frequencies (open symbols) and their arithmetic mean (filled symbols) of various BEDT-TTF salts (see text).

a loss of iodine on the surface of the crystals during the tempering process.

As a result, we state a correlation between the red shift of $\mathrm{CH}_{2}$ vibrational frequencies, i.e., the degree of the interaction between the donor and the anion and the temperatures of the superconducting transitions $T_{c}$. A similar dependence between the size of the unit cell of several $\beta$-phases of BEDT-TTF radical salts and $T_{\mathrm{c}}$ has already been given by Williams et al. [26], which is consistent with the results presented here, because a smaller unit cell should lead to shorter $\mathrm{C}-\mathrm{H}$-anion contacts and therefore to a lower $T_{c}$. Here, we took into account different crystallographic phases with different anions and find the assumption confirmed even for an extended collection of different crystallographic phases of BEDT-TTF radical salts.

From these results it seems that the strength of the interaction between the donor and the acceptor in BEDT-TTF radical salts is a dominating factor ruling the appearance and critical temperature of a superconducting transition. A further indication for this finding arises from the fact that BEDT-TTF salts with strongly electronegative constituents in their anions as $\mathrm{ClO}_{4}^{-}, \mathrm{ReO}_{4}^{-}$and $\mathrm{BF}_{4}^{-}$are not superconductors, simply because these anions will lead to stronger hydrogen bonds to the donor molecules.

\section{Conclusions}

In this paper we present an IR spectroscopic approach to several problems concerning radical salts of BEDTTTF. The assignment of a charge-dependent stretching vibration of the ring $\mathrm{C}=\mathrm{C}$ groups led to the observation of a charge localization in one of the two crystallographic different stacks of $\alpha$-(BEDT-TTF $)_{2} I_{3}$ at the M-I phase 
transition. This should lead to a decrease of dimensionality of the electronic properties in this material, i.e., to a quasi-one-dimensional conductor.

Furthermore, the examination of the $\mathrm{CH}_{2}$ stretching frequencies in BEDT-TTF radical salts containing different anions led us to the conclusion that in salts with a higher $T_{\mathrm{c}}$ the degree of donor-anion interaction is smaller. This is concluded by means of the red shift of these bands, which is due to the hydrogen-bondinglike interaction of the donor with the anion. From these results it would also be interesting to investigate salts with deuterated donors, because it seems likely that an altered donor-anion interaction accounts for such findings as the partially observed inverse isotope effect.

\section{Acknowledgements}

We gratefully acknowledge financial support of this work by the Forschungschwerpunkt Supraleiter des Landes Baden-Württemberg. K.I.P. would like to thank the DAAD for a grant.

\section{References}

1 B. Koch, H.P. Geserich, W. Ruppel, D. Schweitzer, K.H. Dietz and H.J. Keller, Mol. Cryst. Liq. Cryst., 119 (1985) 343.

2 C.S. Jacobsen, J.M. Williams and H.H. Wang, Solid State Commun., 54 (1985) 937.

3 T. Sugano, K. Yamada, G. Saito and M. Kinoshita, Solid State Commun., 55 (1985) 137.

4 M. Meneghetti, R. Bozio and C. Pecile, J. Phys. (Paris), 47 (1986) 1377.

5 A. Ugawa, G. Ojima, K. Yakushi and H. Kuroda, Phys. Rev. B, 38 (1988) 5122 .

6 R. Zamboni, D. Schweitzer, H.J. Keller and C. Taliani, Z. Naturforsch., Teil A, 44 (1989) 295.
7 .S. Gärtner, D. Schweitzer and H.J. Keller, Synth. Met., 44 (1991) 227.

8 J. Moldenhauer, K.I. Pokhodnia, D. Schweitzer and H.J. Keller, Synth. Met, 55-57 (1993) 2554.

9 J. Moldenhauer, Diplomarbeit, Universităt Stuttgart, 1991.

10 M.-H. Whangbo, J.M. Williams, A.J. Schultz, T.J. Emge and M.A. Beno, J. Am. Chem. Soc., 109 (1987) 90.

11 J. Moldenhauer, K.I. Pokhodnia, D. Schweitzer and H.J. Keller, Synth. Met., 55-57 (1993) 2548.

12 R. Bozio, I. Zanon, A. Girlando and C. Pecile, J. Chem. Phys., 71 (1979) 2282.

13 S. Matsuzaki, T. Moriyama and K. Toyoda, Solid State Commun., 34 (1980) 857.

14 M. Meneghetti, R. Bozio, I. Zanon, C. Pecile, C. Ricotta and M. Zanetti, J. Chem. Phys., 80 (1984) 6210.

15 M.E. Kozlov, K.I. Pokhodnia and A.A. Yurchenko, Spectrochim. Acta, Part A, 43 (1987) 323.

16 M.E. Kozlov, K.I. Pokhodnia and A.A. Yurchenko, Spectrochim. Acta, Part A, 45 (1989) 437.

17 M.J. Rice, Solid State Commun., 31 (1979) 93.

18 R. Bozio and C. Pecile, in R.J.H. Clark and R.E. Heester (eds.), Spectroscopy of Advanced Materials, Wiley, New York, 1991.

19 M. Meneghetti and C. Pecile, Phys. Rev. B, 42 (1990) 1605.

20 H. Urayama, H. Yamochi, G. Saito, K. Nozawa, T. Sugano, M. Kinoshita, S. Sato, K. Oshima, A. Kawamoto and J. Tanaka, Chem. Lett., (1988) 55.

21 S. Gärtner, E. Gogu, I. Heinen, H.J. Keller and D. Schweitzer, Solid State Commun., 65 (1988) 1531.

22 A.M. Kini, U. Geiser, H.H. Wang, K.D. Carlson, J.M. Williams, W.K. Kwok, K.G. Vandervoort, J.E. Thompson, D.L. Stupka, D. Jung and M.-H. Whangbo, Inorg. Chem., 29 (1990) 2555.

23 R. Zamboni, D. Schweitzer and H.J. Keller, Synth. Met., 42 (1991) 91.

24 H. Endres, H.J. Keller, R. Swietlik, D. Schweitzer, K. Angermund and C. Krüger, Z. Naturforsch., Teil A, 41 (1986) 1319.

25 Th. Klutz, I. Hennig, U. Haeberlen and D. Schweitzer, Appl. Magn. Res., 2 (1991) 441.

26 J.M. Williams, M.A. Beno, H.H. Wang, U.W. Geiser, T.J. Emge, P.C.W. Leung, G.W. Crabtree, K.D. Carlson, LJ. Azevedo, E.L. Venturini, J.E. Schirber, J.F. Kwak and M.H. Whangbo, Physica, $136 B$ (1986) 371. 